\title{
633 INCORPORATING CHECKPOINT INHIBITORS INTO CANCER CARE: A STUDY OF THE IMPACT OF DIGITAL EDUCATION ON CLINICAL COMPETENCE AND PRACTICE PATTERNS
}

${ }^{1}$ Tariqa Ackbarali*, ${ }^{1}$ Elizabeth del Nido, ${ }^{2}$ Brian Rini, ${ }^{3}$ Michael Overman, ${ }^{3}$ Ignacio Witsuba. ${ }^{1}$ PlatformQ Health Education, Lake Worth, FL, United States; ${ }^{2}$ Vanderbilt-Ingram Cancer Center, Nashville, TN, United States; ${ }^{3}$ MD Anderson Cancer Center, Houston, TX, United States

Background Immune checkpoint inhibitors have transformed the treatment landscape for a variety of tumors and have significantly improved patient prognosis and longevity. Evolving practice standards for diagnostic testing and extensive emerging clinical trial data have left clinicians challenged to apply newer treatments in practice and manage associated side effects. Additionally, improved patient prognosis has created a greater need for survivorship care plans; clinicians must be able to tailor plans to the needs of patients treated with these agents. Education pertaining to biomarker testing, applications of checkpoint inhibitors, adverse event management, and survivorship care is critical to ability to improve patient experience and quality of life.

Methods A 4-hour CME activity was broadcast live-online in June, July, and August 2020 and remained on-demand through February 2021 at OMedLive.com. The program was provided in partnership with the Society for Immunotherapy in Cancer (SITC). The initiative was divided into themes including biomarker usage for checkpoint inhibitor selection, adverse event management, survivorship care, and use of checkpoint inhibitors and combination therapies in the metastatic setting. Knowledge and competence questions were administered pre-, immediate post-, and 2 mos. post-activity. Behavioral impact questions were also asked at follow-up. Data from these questions were analyzed to determine engagement and clinical impact.

Results Final program results from 1,909 learners showed that post-activity engagement resulted in $61 \%$ reporting a positive impact on patient experience, and $74 \%$ reporting a positive impact on clinical practice, with 179 qualitative write-in examples detailing improvements in diagnosis, use of newer therapies, ability to manage adverse events, and patients' tolerance of treatments. All $14 \mathrm{CME}$ test questions reflected statistically significant improvements on biomarker utility, checkpoint inhibitors, combination therapy applications, adverse event management, and survivorship care, with an average of $15 \%$ pre to 2-month follow-up improvement. The overall average effect size from pre- to post-test was $\mathrm{d}=1.27$, and $\mathrm{d}=$ 0.429 for pre- to 2-month follow-up point. Practice pattern questions elucidated preferences for biomarker testing, challenges of integrating immunotherapy, areas of difficulty in survivorship care, and challenges enrolling in clinical trials.

Conclusions The activity was successful in improving clinician understanding of the use of biomarker testing to determine treatment plans, applications of checkpoint inhibitors and combination therapies, adverse event management, and survivorship care planning. Open-ended responses to behavioral impact questions illustrated clear improvements in clinicianreported patient impacts, including improved psychological tolerance of treatment, quality of life, and overall wellness.

http://dx.doi.org/10.1136/jitc-2021-SITC2021.633 